\title{
Transjugular Intrahepatic Portosystemic Shunt for Acute Variceal Bleeding in Patients with Viral Liver Cirrhosis: Predictors of Early Mortality
}

Chang Jin Yoon ${ }^{1}$

Jin Wook Chung

Jae Hyung Park
DOI:10.2214/AJR.04.0607

Received April 19, 2004; accepted after revision October 15, 2004.

${ }^{1}$ All authors: Department of Radiology and Institute of Radiation Medicine, Seoul National University College of Medicine, Clinical Research Institute, 28 Yongon-Dong, Chongno-Gu, Seoul 110-774, South Korea. Address correspondence to J.W. Chung

(chungjw@radcom.snu.ac.kr).

AJR 2005;185:885-889

0361-803X/05/1854-885

(C) American Roentgen Ray Society

OBJECTIVE. The purpose of our study was to assess the predictors of early mortality after the creation of transjugular intrahepatic portosystemic shunts (TIPS) for acute variceal bleeding in patients with viral liver cirrhosis.

MATERIALS AND METHODS. Seventy-three patients (56 men and 17 women; mean age, 51.3 years) with viral liver cirrhosis who underwent TIPS placement for acute variceal bleeding were studied. Multiple covariates, including demographic, clinical, and biochemical parameters, were included in univariate and multivariate analyses to determine their association with early (30-day) mortality.

RESULTS. During the follow-up period (mean, 35 months 3 days), shunt dysfunction occurred in 33 patients (45.2\%). Forty-three patients (58.9\%) died, and 23 patients $(31.5 \%)$ died within 30 days of TIPS. Early death was predicted independently by hyperbilirubinemia ( $>3$ $\mathrm{mg} / \mathrm{dL} ; p=0.004$; odds ratio, 10.6$)$ and elevated serum creatinine level $(>1.7 \mathrm{mg} / \mathrm{dL} ; p=0.018$; odds ratio, 12.0).

CONCLUSION. Hyperbilirubinemia and elevated serum creatinine level are predictive of early mortality after TIPS creation for acute variceal bleeding in patients with viral liver cirrhosis.


he transjugular intrahepatic portosystemic shunt (TIPS) is a well-established therapy for patients with liver cirrhosis complicated by acute or recurrent variceal bleeding that is refractory to endoscopic therapy and intractable ascites [1-4]. However, TIPS are associated with high early mortality; the 30-day mortality rate ranges from $25 \%$ to $30 \%$ [1-4]. Many authors have investigated prognostic factors accurately predicting survival after TIPS placement [5-13]. Several factors predictive of poor survival have been recognized: hyponatremia, hyperbilirubinemia, elevated serum alanine aminotransferase, advanced liver disease, emergent TIPS placement, and coexisting renal insufficiency [5-13].

To date, most studies about TIPS have been from Western countries, and most patients included in these studies had alcoholic liver cirrhosis [1-13]. However, in Asian countries, hepatitis virus carriers are reported to be $5-20 \%$ of the population, and viral hepatitis is the main cause of liver cirrhosis [14, 15]. Viral liver cirrhosis is different from alcoholic liver cirrhosis in many aspects such as histologic and morphologic changes, disease progression, treatment options, and incidence of hepatocellular carcinoma (HCC) [16-18]. Therefore, it is inappropriate to apply the Western data to patients with viral liver cirrhosis, especially in hepatitis virus-endemic areas. However, a few studies from Asian countries have investigated prognostic factors of TIPS in patients with viral liver cirrhosis. We performed this study to assess the prognostic factors for early mortality after TIPS placement for acute variceal bleeding in patients with viral liver cirrhosis. To our knowledge, this is the first English-language article from a hepatitis virus-endemic area that describes the prognostic factors after TIPS placement.

\section{Materials and Methods Study Population}

This retrospective study was approved by the review board of our institution. The medical records of 111 consecutive patients who underwent TIPS placement in our institution between June 1991 and January 2002 were reviewed to verify the cause of liver disease and indication for TIPS and to obtain all relevant clinical and laboratory data. Thirty-eight patients were excluded because of their cause of liver cirrhosis (alcoholic $[n=23]$ and cholestatic $[n=1]$ liver disease) or elective TIPS (recurrent variceal 
bleeding $[n=10]$ and intractable ascites $[n=4])$. Emergent indications for TIPS was defined as a patient requiring blood products within $24 \mathrm{hr}$ of TIPS creation, hemodynamic instability, balloon tamponade, or obvious continued bleeding [6].

The patient cohort consisted of 56 men and 17 women with a mean age of 51.3 years (range, 27-85 years). The causes of liver disease were hepatitis $\mathrm{B}(n=63)$ and hepatitis $\mathrm{C}(n=10)$ virus. According to the modified Child-Pugh classification, 15 patients had class A liver disease, 20 patients class $\mathrm{B}$, and 35 patients class $\mathrm{C}$. The remaining three patients had insufficient data to classify them. Ten patients had documented HCC before TIPS creation that had been treated with transcatheter arterial chemoembolization (TACE, $n=9$ ) or percutaneous ethanol injection $(n=1)$. In four patients, HCC was detected on pre-TIPS CT and treated with TACE within 7 days after TIPS placement.

\section{TIPS Procedure}

TIPS were created using a technique described elsewhere [1-4]. The TIPS was usually created between the right hepatic vein and the right portal vein $(n=40)$, or, in patients with a small right hepatic vein, between the middle hepatic and the right portal veins $(n=17)$. In 16 cases, the shunt was created between the middle hepatic and the left portal veins $(n=10)$ or between the left hepatic and left portal veins $(n=6)$ because of right portal vein thrombosis $(n=8)$ or an unfavorable location of the HCC $(n=8)$. Shunt tracts were dilated with 10 -mm angioplasty balloons (Boston Scientific/Medi-Tech) and lined with various commercially available stents: Wallstents (Boston Scientific/Medi-Tech, $n=49$ ), Niti-S (Taewoong, $n=17$ ), Memotherm (Angiomed, $n=4$ ), and Gianturco Z stent (Cook, $n=3$ ). Stents of 10-mm-diameter $(n=55)$ and 8-mm-diameter $(n=10)$ were used. Stent size was not recorded in the remaining eight patients. The goal of the procedure was to reduce the portosystemic pressure gradient (PPG, measured between the portal vein and right atrium) to less than $12 \mathrm{~mm} \mathrm{Hg}$, which was achieved in 45 of 69 patients (four patients did not have hemodynamic measurements recorded).

\section{Follow-Up}

Doppler sonography was performed before discharge, at 3-4 months intervals, and whenever shunt dysfunction was suspected clinically. Shunt dysfunction was suspected on Doppler sonography if the calculated peak velocity in the shunt was less than $60 \mathrm{~cm} / \mathrm{sec}$ or reversal of flow was shown on color flow imaging of the draining hepatic vein or portal vein [19]. Results from Doppler sonography were confirmed with angiography. Stenosis was defined on angiography if there was a $50 \%$ or greater narrowing of the lumen or the portosystemic gradient was $12 \mathrm{~mm} \mathrm{Hg}$ or greater.

Patients were followed up from their date of TIPS placement until death, liver transplantation, or study closure. The date of the last clinic visit or liver transplantation defined the follow-up period for all surviving patients. If no clinic visits were recorded in the medical records, the patients or their families were contacted by telephone $(n=9)$ and a brief interview was conducted to determine the patient's clinical course after TIPS creation.

\section{Statistical Analysis}

Sixteen variables related to clinical or biochemical data were included in the analysis. Continuous variables were dichotomized on the basis of the existing literature [10-12]: albumin level $(<2.8$ vs $\geq 2.8$ $\mathrm{mg} / \mathrm{dL}$ ), prothrombin time (PT; $<60 \mathrm{vs} \geq 60 \%$ ), total bilirubin level ( $>3 \mathrm{vs} \leq 3 \mathrm{mg} / \mathrm{dL}$ ), serum creatinine level (>1.7 vs $\leq 1.7 \mathrm{mg} / \mathrm{dL}$ ), alanine aminotransferase (ALT) level ( $>70$ vs $\leq 70 \mathrm{IU} / \mathrm{L}$ ), aspartate aminotransferase (AST) level (> 70 vs $\leq 70 \mathrm{IU} / \mathrm{L}$ ), WBC (>8 vs $\leq 8 \times 10^{3} / \mu \mathrm{L}$ ), platelet count (>120 vs $\leq 120 \times 10^{3} / \mu \mathrm{L}$ ), and PPG before ( $>18$ vs $\leq 18 \mathrm{~mm}$ $\mathrm{Hg}$ ) and after ( $>12 \mathrm{vs} \leq 12 \mathrm{~mm} \mathrm{Hg}$ ) TIPS placement. Categoric variables included sex, cause of liver disease (hepatitis B vs C), comorbid conditions (hypertension, diabetes, and HCC; presence vs absence), and route of TIPS (right portal vein vs left portal vein).

The univariate association between individual prognostic factors and early mortality (30 days) was tested using Fisher's exact test or the Student's $t$ test as appropriate, and multiple logistic regression was used to test the significance of factors adjusted for one another. For all analyses, a $p$ value of less than 0.05 and a $95 \%$ confidence interval that did not span unity were considered the thresholds of statistical significance.

Patient data were censored at time of death, liver transplantation, or last follow-up. Kaplan-Meier analyses were performed to estimate time to death, and the log-rank test was used to determine whether differences were significant. All analyses were conducted with SPSS for Windows (Microsoft) version 10.0 software (SPSS).

\section{Results}

\section{Outcomes of TIPS Placement}

TIPS were successfully created in all the 73 patients. The characteristics of the patients are shown in Table 1. The mean pre- and post-TIPS portosystemic pressure gradients were 26.5 and $10.2 \mathrm{~mm} \mathrm{Hg}$, respectively. Variceal bleeding stopped in $61(83.6 \%)$ of 73 patients after creation of the TIPS, and 12 patients continued to bleed. In the 12 patients, shunt occlusions due to acute thrombosis were detected in four patients but they refused further treatment. In the remaining eight patients, the shunts were patent on Doppler sonography. One patient underwent splenorenal shunt surgery, and the remaining 11 patients died of persistent bleeding. In one patient, hemoperitoneum developed after TIPS placement that contributed to accelerated multiorgan failure, and the patient died 2 days after TIPS placement.

The mean and median times of follow-up were 35 months 3 days and 23 months 6 days (range, 2 days-136 months). Shunt dysfunction occurred in 33 patients during the followup period. Shunt occlusion due to acute thrombosis occurred in nine patients. Five patients were successfully treated with aspiration thrombectomy and balloon angioplasty. The remaining four patients refused further intervention. Shunt stenoses developed in 24 patients, which were detected by rebleeding $(n=13)$, Doppler sonography $(n=9)$, and recurrent ascites $(n=2)$. Rebleeding developed within 6 months in seven patients (29.2\%) and within 12 months in 12 patients $(n=50 \%)$. Fifteen patients underwent percutaneous shunt revision with balloon angioplasty. Additional stents were required in six patients for sufficient coverage of the hepatic venous end of the shunt tract. Primary and secondary patency rates of the TIPS tract were $61.3 \%$ and $76.4 \%$ at 1 year, $49.3 \%$ and $72.0 \%$ at 2 years, and $42.9 \%$ and $68.9 \%$ at 3 years, respectively.

Forty-three patients died during the follow-up period; $23(31.5 \%)$ of them died within the first 30 days after TIPS placement. The causes of 30-day mortality included uncontrolled bleeding $(n=11)$, multiorgan system failure $(n=7)$, liver failure $(n=3)$, renal failure $(n=1)$, and adult respiratory distress syndrome $(n=1)$. The causes of death beyond 30 days were progressive liver failure $(n=7)$ followed by sepsis with shock $(n=4)$, recurrent variceal bleeding $(n=3)$, renal failure $(n=3)$, gastric ulcer bleeding $(n=1)$, and unknown $(n=2)$. Overall survival rates were $71.1 \%$ at 1 year, $61.2 \%$ at 2 years, $49.5 \%$ at 3 years, and $35.4 \%$ at 5 years.

\section{Determinants of Early Mortality}

In univariate analyses, serum albumin level, total bilirubin level, PT, and serum creatinine level were associated with 30-day mortality (Table 2). The 30-day mortality rate of patients with serum albumin level $<2.8 \mathrm{mg} / \mathrm{dL}$ was $46.2 \%(30 / 65)$ versus $13.8 \%$ (9/65) for $\geq 2.8$ $\mathrm{mg} / \mathrm{dL} \quad(p=0.019)$. In patients with a PT $<60 \%$, the 30-day mortality rate was $55.7 \%(34 / 61)$ compared with $14.8 \%(9 / 61)$ in 


\section{Early Mortality with TIPS for Variceal Bleeding}

patients with a PT of $\geq 60 \% \quad(p=0.001)$. Thirty-day mortality rates in patients with total bilirubin levels $>3$ and $\leq 3 \mathrm{mg} / \mathrm{dL}$ were $56.5 \%$ $(13 / 23)$ and $20 \% \quad(9 / 45)$, respectively $(p<0.001)$. The 30 -day mortality rates in patients with creatinine levels $>1.7$ and $\leq 1.7$ $\mathrm{mg} / \mathrm{dL}$ were $55.6 \%(10 / 18)$ and $23.4 \%(11 / 47)$ $(p<0.001)$. In multivariate analysis, only two variables retained independent predictive value: total bilirubin level and creatinine level (Table 3). Figures 1 and 2 show Kaplan-Meier survival estimates between patients with hyperbilirubinemia and the remaining group ( $p=0.001$ by log-rank test) and between patients with elevated creatinine level and the remaining group ( $p=0.001$ by log-rank test).

\section{Discussion}

In the past decade, TIPS placement has become a widely used, validated therapy for patients with portal hypertension [1-4]. Although accepted indications of TIPS include recurrent variceal bleeding, portal hypertensive gastropathy, refractory ascites or hydrothorax, and Budd-Chiari syndrome, acute variceal bleeding is the most established indication, with little debate about TIPS efficacy [1-4].

When TIPS is used as rescue therapy in acute variceal bleeding, early (1-month) mortality rates range from $28 \%$ to $48 \%$, which are significantly higher than those after elective TIPS placement (11-26\%) [5-13]. Although numerous studies have evaluated factors that affect patient survival after elective TIPS placement [5-9], only three articles have analyzed predictors of early death after emergent TIPS placement for acute variceal bleeding [10-12]. Banares et al. [10] found that hepatic encephalopathy before TIPS placement, ascites before TIPS placement, and a serum albumin level lower than $2.7 \mathrm{~g} / \mathrm{L}$ were independently related to 30 -day mortality. Sanyal et al. [11] reported that aspiration and grade IV encephalopathy were independent predictors of early death. Finally, Patch et al. [12] found six variables were related to early mortality: moderate or severe ascites, need for ventilation, WBC, platelet count, partial thromboplastin time, and creatinine. Those authors obtained a prognostic index score combining the six variables that was prospectively validated in another series of patients [12]. However, evaluation of encephalopathy and ascites is somewhat subjective and likely to be affected by concomitant medical therapies. Therefore, we did not include these factors in the analyses for prognostic factors. For the same reason, individual components of Child-Pugh classifica- tion were included in the analyses instead of the classification itself.

In our study, elevated serum bilirubin and creatinine levels were independent predictors

TABLE I: Demographic, Clinical, and Biochemical Characteristics of Study Population

\begin{tabular}{|c|c|c|c|}
\hline Characteristic & No. & $\%$ & Mean (SD) \\
\hline \multicolumn{4}{|l|}{ Demographic } \\
\hline Age (yr) & $73^{\mathrm{a}}$ & 100.0 & $51.3(9.9)$ \\
\hline Men & 56 & 76.7 & \\
\hline \multicolumn{4}{|l|}{ Cause of cirrhosis } \\
\hline Hepatitis $B$ virus & 63 & 86.3 & \\
\hline Hepatitis C virus & 10 & 13.7 & \\
\hline Child-Pugh score & $63^{\mathrm{a}}$ & 86.3 & $9.1(3.0)$ \\
\hline \multicolumn{4}{|l|}{ Child-Pugh classification } \\
\hline A & 15 & 20.5 & \\
\hline B & 20 & 27.4 & \\
\hline C & 35 & 47.9 & \\
\hline \multicolumn{4}{|l|}{ Comorbid conditions } \\
\hline Hypertension & 13 & 17.8 & \\
\hline Diabetes & 10 & 13.7 & \\
\hline Hepatocellular carcinoma & 13 & 17.8 & \\
\hline \multicolumn{4}{|l|}{ Biochemical } \\
\hline Albumin $(\mathrm{g} / \mathrm{dL})$ & $65^{\mathrm{a}}$ & 89.0 & $2.7(0.5)$ \\
\hline AST (IU/L) & $55^{a}$ & 75.3 & $59.7(10.5)$ \\
\hline ALT (IU/L) & $55^{\mathrm{a}}$ & 75.3 & $43.5(11.4)$ \\
\hline Total bilirubin (mg/dL) & $68^{\mathrm{a}}$ & 93.2 & $3.9(6.4)$ \\
\hline Prothrombin (\%) & $61^{\mathrm{a}}$ & 83.6 & $60.4(17.6)$ \\
\hline $\mathrm{WBC}\left(\times 10^{3} / \mu \mathrm{L}\right)$ & $59^{a}$ & 80.8 & $8.1(2.1)$ \\
\hline Platelet count $\left(\times 10^{3} / \mu \mathrm{L}\right)$ & $60^{\mathrm{a}}$ & 82.2 & $120(32)$ \\
\hline Serum creatinine $(\mathrm{mg} / \mathrm{dL})$ & $65^{\mathrm{a}}$ & 89.0 & $2.7(1.5)$ \\
\hline \multicolumn{4}{|l|}{ PPG $(\mathrm{mmHg})$} \\
\hline Before TIPS & $65^{\mathrm{a}}$ & 89.0 & $26.5(6.5)$ \\
\hline After TIPS & $65^{a}$ & 89.0 & $10.2(3.1)$ \\
\hline
\end{tabular}

Note-SI unit conversion: albumin and hemoglobin, $\times 10 \mathrm{~g} / \mathrm{L}$; bilirubin, $\times 17.1 \mu \mathrm{mol} / \mathrm{L} ;$ creatinine,$\times 88.4 \mu \mathrm{mol} / \mathrm{L}$. PPG $=$ portosystemic pressure gradient (measured between portal vein and right atrium), TIPS $=$ transjugular intrahepatic portosystemic shunt, AST = aspartate aminotransferase, ALT = alanine aminotransferase. ${ }^{a}$ Number of patients available for each variable.

TABLE 2: Univariate Analysis for Early Mortality After Creation of Transjugular Intrahepatic Portosystemic Shunt

\begin{tabular}{l|c|c|c}
\hline \multicolumn{1}{c|}{ Predictor of Early Mortality } & $p$ & Odds Ratio & $95 \% \mathrm{Cl}$ \\
\hline Serum albumin, $<2.8 \mathrm{vs} \geq 2.8 \mathrm{~g} / \mathrm{dL}$ & 0.019 & 5.152 & $1.311-20.245$ \\
Prothrombin, $<60 \%$ vs $\geq 60 \%$ & 0.001 & 7.362 & $2.149-25.215$ \\
Total bilirubin, $>3 \mathrm{vs} \leq 3 \mathrm{mg} / \mathrm{dL}$ & 0.007 & 5.041 & $1.582-16.270$ \\
Serum creatinine, $>1.7 \mathrm{vs} \leq 1.7 \mathrm{mg} / \mathrm{dL}$ & 0.009 & 10.200 & $1.775-58.623$ \\
\hline
\end{tabular}

Note- $\mathrm{Cl}=$ confidence interval.

TABLE 3: Multivariate Analysis for Early Mortality After Creation of Transjugular Intrahepatic Portosystemic Shunt

\begin{tabular}{l|c|c|c}
\hline Independent Predictor of Early Mortality & $p$ & Odds Ratio & $95 \% \mathrm{Cl}$ \\
\hline Total bilirubin $>3 \mathrm{mg} / \mathrm{dL}$ & 0.004 & 10.609 & $2.082-54.056$ \\
Serum creatinine $>1.7 \mathrm{mg} / \mathrm{dL}$ & 0.018 & 11.997 & $1.526-94.345$ \\
\hline
\end{tabular}




\section{Yoon et al.}

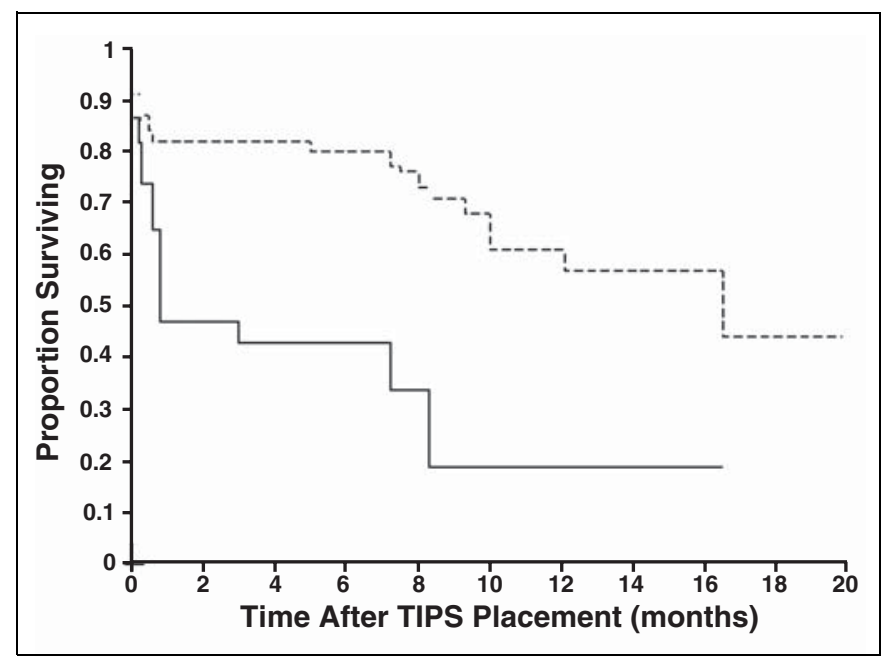

Fig. 1-Kaplan-Meier survival curves based on serum bilirubin level show significantly higher mortality rate in patients with hyperbilirubinemia $(>3 \mathrm{mg} / \mathrm{dL}$ $[n=23]$, solid line) ( $p=0.001$ by log-rank test) than in those without. Dotted line indicates bilirubin level $\leq 3 \mathrm{mg} / \mathrm{dL}(n=45)$.

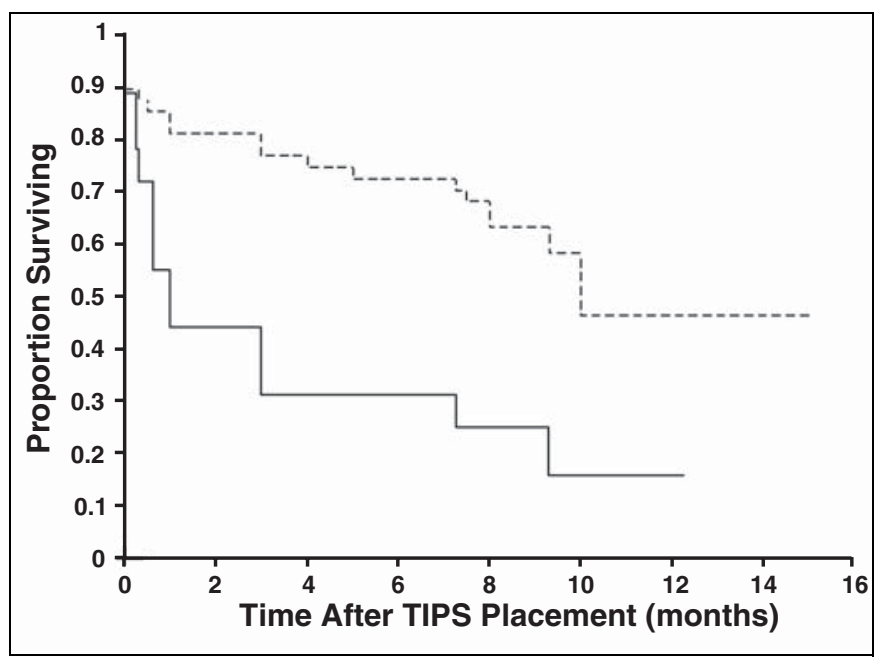

Fig. 2-Kaplan-Meier survival curves based on serum creatinine level show significantly higher mortality rate in patients with elevated serum creatinine level ( $>1.7 \mathrm{mg} / \mathrm{dL}[n=18]$, solid line) ( $p=0.001$ by log-rank test) than in those without. Dotted line indicates serum creatinine level $\leq 1.7 \mathrm{mg} / \mathrm{dL}, n=47$. of early death. In patients with bilirubin $>3$ $\mathrm{mg} / \mathrm{dL}$ and creatinine $>1.7 \mathrm{mg} / \mathrm{dL}$, the risk of early death was 10.6 and 12.0 times greater than in patients with bilirubin $\leq 3 \mathrm{mg} / \mathrm{dL}$ and creatinine $\leq 1.7 \mathrm{mg} / \mathrm{dL}$, respectively. Patients with these risk factors had a very high rate of early mortality.

Hyperbilirubinemia has been a frequently designated predictor of early mortality in other studies. Rajan et al. [6], after reviewing 220 patients, reported independent association of the total bilirubin level with early mortality, with the odds ratios of 4.4 in acute variceal bleeding and 7.1 in recurrent bleeding, respectively. Chalasani et al. [7] found that total bilirubin level $\geq 3.0 \mathrm{mg} / \mathrm{dL}$ had an independent effect on 30-day mortality (relative risk, 5.4). That study included patients who had TIPS placed for variceal bleeding and those who had TIPS placed for intractable ascites. These results are not astonishing because the serum bilirubin level reflects hepatic synthetic dysfunction in chronic and acutely diseased metabolic states.

In our study, elevated serum creatinine level was another independent factor of early mortality after TIPS placement. The association of an elevated creatinine level with poor survival has been suggested in both emergent and elective TIPS placement $[5,12,13]$. Patch et al. [12] proposed that an elevated serum creatinine level is independently associated with early mortality after TIPS placement in patients with acute variceal bleeding. Russo et al.
[13], in a multivariate analysis of outcomes of TIPS placement for variceal bleeding and intractable ascites, reported elevated serum creatinine level was the strongest predictor of 30day mortality. Therefore, we think that elevated serum bilirubin and creatinine levels are important predictors of early mortality in both emergent and elective TIPS placement.

In our study, $31.5 \%$ (23/73) patients died within 1 month after TIPS creation. The most common cause of early mortality was persistent variceal bleeding (11/23 patients) despite successful TIPS creation. Ten of the 11 patients had hyperbilirubinemia (>3 mg/dL), and eight had an elevated serum creatinine level $(>1.7 \mathrm{mg} / \mathrm{dL})$. In these patients, therefore, decompression of portal hypertension is insufficient, and embolization of the varix itself may be needed for immediate control of variceal bleeding. We had four early deaths in patients with acute thrombotic shunt occlusions. Some investigators speculated that the influx of bile into the shunt may stimulate thrombosis and suggested the use of a covered stent-graft to avoid direct bile-shunt contact $[20,21]$. In a recent study in which a polytetrafluoroethylene-covered stent-graft was used for the TIPS, only one early shunt occlusion occurred in 71 patients [22].

Recently, in Western countries, TIPS placement has been used in candidates for liver transplantation as a bridge to transplantation [23, 24]. TIPS placement may prolong the waiting period in such candidates and fa- cilitate surgery by the reduction of portal pressure and obliteration of collaterals. However, because liver transplantation has not yet been widely performed in Asia [25], TIPS has been accepted in Asian countries as a definite and permanent treatment for portal decompression. Therefore, precise prediction and meticulous survey for TIPS dysfunction are no less important than those for early mortality in Asian countries.

Since Malinchoc et al. [26] developed a model of end-stage liver disease (MELD) to predict the 3-month survival rate after TIPS placement, several recent studies indicated that early mortality after elective TIPS placement could be accurately predicted using the MELD score [27-29]. Originally, the MELD score included the cause of liver cirrhosis as an individual component because the outcomes of patients with cholestatic or alcoholic liver cirrhosis were better than for those with viral hepatitis. It has been suggested that viral hepatitis has a more rapidly progressive course, or that patients with viral hepatitis have more impaired hepatocellular function and are less tolerant to portosystemic shunting [26]. However, recently several authors reported that the cause of liver disease has little effect on patient survival and deleted cause of liver disease in the modification of the MELD score [8, 9, 29]. Those studies divided patients into subgroups of those with alcoholic and those with nonalcoholic liver disease; nonalcoholic liver disease included hepatitis B viral, hepatitis C vi- 


\section{Early Mortality with TIPS for Variceal Bleeding}

ral, cholestatic, and cryptogenic liver disease $[8,9,29]$. Therefore, the number of patients with viral liver disease, especially hepatitis B, was relatively small and accounted for $4.2-7.2 \%$ of the total population of the studies $[8,9]$. In a recent investigation from an area in which hepatitis B virus is endemic, which included similar numbers of patients with alcoholic and viral liver disease, the patients with viral liver disease experienced more severe deterioration of liver function after TIPS placement [30]. Therefore, we think that the application of previously published data to patients with viral liver cirrhosis, especially in hepatitis endemic areas, is inappropriate. Further investigation comparing the prognosis after TIPS placement between patients with viral liver cirrhosis and those with alcoholic liver cirrhosis is needed.

In our group of patients with viral liver cirrhosis complicated by acute variceal bleeding, hyperbilirubinemia and an elevated creatinine level were associated with an increased risk of early mortality after TIPS placement.

\section{References}

1. Rossle M, Haag K, Ochs A, et al. The transjugular intrahepatic portosystemic stent-shunt procedure for variceal bleeding. $N$ Engl J Med 1994; 330:165-171

2. Ochs A, Rossle M, Haag K, et al. The transjugular intrahepatic portosystemic stent-shunt procedure for refractory ascites. N Engl J Med 1995; 332:1192-1197

3. Blokzijl H, de Knegt RJ. Long-term effect of treatment of acute Budd-Chiari syndrome with a transjugular intrahepatic portosystemic shunt. Hepatology 2002; 35:1551-1552

4. Mezawa S, Homma H, Ohta H, et al. Effect of transjugular intrahepatic portosystemic shunt formation on portal hypertensive gastropathy and gastric circulation. Am J Gastroenterol 2001; 96:1155-1159

5. Jalan R, Elton RA, Redhead DN, Finlayson ND, Hayes PC. Analysis of prognostic variables in the prediction of mortality, shunt failure, variceal rebleeding and encephalopathy following the TIPS for variceal hemorrhage. J Hepatol 1995; 23: $123-128$

6. Rajan DK, Haskal ZJ, Clark TWI. Serum bilirubin and early mortality after transjugular intrahepatic portosystemic shunt: results of a multivariate anal- ysis. J Vasc Interv Radiol 2002; 13:155-161

7. Chalasani N, Clark WS, Martin LG, et al. Determinants of mortality in patients with advanced cirrhosis after TIPS. Gastroenterology 2000; 118:138-144

8. Ferral H, Gamboa P, Postoak DW, et al. Survival after elective transjugular intrahepatic portosystemic shunt creation: prediction with model for end-stage liver disease score. Radiology 2004; 231:231-236

9. Ferral H, Vasan R, Speeg KV, et al. Evaluation of a model to predict poor survival in patients undergoing elective TIPS procedures. J Vasc Interv Radiol 2002; 13:1103-1108

10. Banares R, Casado M, Rodriguez-Laiz JM, et al. Urgent transjugular intrahepatic portosystemic shunt for control of acute variceal bleeding Am J Gastroenterol 1998; 93:75-79

11. Sanyal AJ, Freedman AM, Luketic VA, et al. The natural history of portal hypertension after transjugular intrahepatic portosystemic shunt. Gastroenterology 1997; 112:889-898

12. Patch D, Nikolopoulou V, McCormick A, et al. Factors related to early mortality after transjugular intrahepatic portosystemic shunt for failed endoscopic therapy in acute variceal bleeding. J Hepatol 1998; 28:454-460

13. Russo MW, Jacques PF, Mauro M, Odell P, Brown RS. Predictors of mortality and stenosis after transjugular intrahepatic portosystemic shunt. Liver Transpl 2002; 8:271-277

14. Margolis HS, Alter MJ, Hadler SC. Hepatitis B: evolving epidemiology and implications for control. Semin Liver Dis 1991; 11:84-92

15. Lee HS, Kim CY. Seroepidemiology of HBV and $\mathrm{HCV}$ in Korea: the decreasing prevalence rate of $\mathrm{HBV}$ infection after launching HB vaccination program. International Hepatology Communications 1996; 5:53-61

16. Herold C, Regn S, Ganslmayer M, et al. Can quantitative tests of liver function discriminate between different etiologies of liver cirrhosis? Dig Dis Sci 2002; 47:2669-2673

17. Vizzotto L, Vertemati M, Gambacorta M, et al. Analysis of histological and immunohistochemical patterns of the liver in posthepatitic and alcoholic cirrhosis by computerized morphometry. Mod Pathol 2002; 15:798-806

18. Tiribelli C, Melato M, Croce LS, et al. Prevalence of hepatocellular carcinoma and relation to cirrhosis: comparison of two different cities of the world-Trieste, Italy, and Chiba, Japan. Hepatology 1989; 10:998-1002

19. Foshagaer MC, Ferral H, Nazarian GK, Cas-
taneda-Zuniga WR, Letourneau JG. Duplex sonography after transjugular intrahepatic portosystemic shunts: normal hemodynamic findings and efficacy in predicting shunt patency and stenosis. AJR 1995; 165:1-7

20. Saxon RR, Mendel-Hartvig J, Corless CL, et al. Bile duct injury as a major cause of stenosis and occlusion in transjugular intrahepatic portosystemic shunts: comparative histopathologic analysis in humans and swine. J Vasc Interv Radiol 1996; 7:487-497

21. Cejna M, Peck-Radosavljevic M, Thurnher SA, Hittmair K, Schoder M, Lammer J. Creation of transjugular intrahepatic portosystemic shunts with stent-grafts: initial experience with a polytetrafluoroethylene-covered nitinol endoprosthesis. Radiology 2001; 221:437-446

22. Hausegger KA, Karnel F, Georgieva B, et al. Transjugular intrahepatic portosystemic shunt creation with the Viatorr expanded polytetrafluoroethylenecovered stent-graft. J Vasc Interv Radiol 2004; 15:239-248

23. Moreno A, Meneu JC, Moreno E, et al. Liver transplantation and transjugular intrahepatic portosystemic shunt. Transplant Proc 2003; 35:1869-1870

24. Riggio O, Angeloni S, Merli M, et al. TIPS for patients awaiting orthotopic liver transplantation. Liver Transpl 2003; 9:999-1000

25. Park KM, Lee SG, Lee YJ, et al. Adult-to-adult living donor liver transplantation at Asian Medical Center, Seoul, Korea. Transplant Proc 1999; 31:456-458

26. Malinchoc M, Kamath PS, Gordon FD, Peine CJ, Rank J, ter Borg PC. A model to predict poor survival in patients undergoing TIPS. Hepatology 2000; 31:864-871

27. Schepke M, Roth F, Fimmers R, et al. Comparison of MELD, Child-Pugh, and Emory model for the prediction of survival in patients undergoing transjugular intrahepatic portosystemic shunting. Am J Gastroenterol 2003; 98:1167-1174

28. Salerno F, Merli M, Cazzaniga M, et al. MELD score is better than Child-Pugh score in predicting 3-month survival of patients undergoing transjugular intrahepatic portosystemic shunt. J Hepatol 2002; 36:494-500

29. Kamath PS, Wiesner RH, Malinchoc M, et al. A model to predict survival in patients with end-stage liver disease. Hepatology 2001; 33:464-470

30. Song HG, Lee HC, Park YH, et al. Therapeutic efficacy of transjugular intrahepatic portosystemic shunt on bleeding gastric varices [in Korean]. Taehan Kan Hakhoe Chi 2002; 8:448-457 